\title{
Photo-triggered Aggregation-Induced Emission and Direct Generation of 4D Soft Patterns
}

Huilin Xie, ${ }^{\dagger}$ Zhao Li,,${ }^{*} \S$ Junyi Gong, ${ }^{\dagger}$ Lianrui Hu, ${ }^{\dagger}$ Parvej Alam, ${ }^{\dagger}$ Xiaofan Ji, ${ }^{\dagger}$ Yubing Hu, ${ }^{\dagger}$

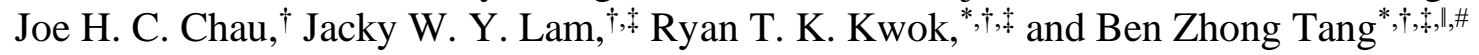

†Department of Chemistry, Hong Kong Branch of Chinese National Engineering Research Center for Tissue Restoration and Reconstruction, The Hong Kong University of Science and Technology (HKUST), Clear Water Bay, Kowloon, Hong Kong, China

${ }^{\S}$ Institute of Engineering Medicine, Beijing Institute of Technology, 5 South Zhongguancun Street, Haidian District, Beijing 100081, China

†HKUST Shenzhen Research Institute, No. 9 Yuexing 1st RD, South Area Hi-Tech Park, Nanshan, Shenzhen 518057, China

"Shenzhen Institute of Molecular Aggregate Science and Engineering, School of Science and Engineering, The Chinese University of Hong Kong, Shenzhen, 2001 Longxiang Boulevard, Longgang District, Shenzhen City, Guangdong 518172, China

\#State Key Laboratory of Luminescent Materials and Devices, and Center for AggregationInduced Emission (Guangzhou International Campus), South China University of Technology, Guangzhou 510640, China

Keywords: photo-triggered aggregation-induced emission, gel, spatio-temporal control, selfhealing, laser-mediated mask-free writing, 4D soft patterns

\section{Abstract text}

The microscopic control of macroscopic phenomena is one of the core subjects in materials science. In particular, the spatio-temporal control of material behaviors through a non-contact way is of fundamental importance but different to accomplish. Herein, a strategy to realize remote spatio-temporal control of luminescence behaviors is reported. A multi-arm salicylaldehyde benzoylhydrazone-based aggregation-induced emission luminogen (AIEgen)/metal ion system was developed of which the fluorescence can be gated by the UV irradiation with time dependency. By changing metal ion species, the fluorescence can also be tuned. The mechanism of the UV-mediated fluorescence change behaivors was investigated and it was revealed that a photo-triggered aggregation-induced emission (PTAIE) process dominates the behaviors. The AIEgen was further covalently integrated into a polymeric network and the formed gel/metal ion system can achieve laser-mediated mask-free writing 
enabled by the PTAIE process. Moreover, by further taking advantage of the time-dependent self-healing property of hydrazone-based dynamic covalent bond, transformable fourdimensional (4D) soft patterns were generated. The findings and the strategy increase the choice of ways to manipulate molecules on the supramolecule or aggregate level. They also show opportunities for the development of controllable smart materials and expand the scope of the materials in advanced optoelectronic applications.

\section{Introduction}

Matter is a physical substance that exists in solid, liquid, or gaseous state. It is typically made up of microscopic particles and behaves differently in all three states. ${ }^{[1]}$ While the phase transition of the matter is a macroscopic process that is manifested by the appearance of new properties of the matter. ${ }^{[2]}$ Interestingly, these macroscopic processes are often accompanied by the transformation of different states of the same substance. ${ }^{[3]}$ For example, rivers turn to be ice in cold winter, and the ice melts in spring. However, these natural changes are mostly induced by modification in atomic or molecular arrangements and can be controlled by additional stimulations such as temperature and pressure. ${ }^{[2]}$ The phase transition, which emerged in distinct ordered two phases across the critical region, ${ }^{[4]}$ is of vital significance in materials science. ${ }^{[5]}$ In 1947 , W. M. Winslow first discovered a phase-changing material that is electrorheological (ER) fluid. Its viscosity can be changed under the electric field stimulation. ${ }^{[6]}$ This macroscopic transition process is very hard to control locally and precisely because the phase change is the inherently collective behavior of the suspension mediums. ${ }^{[7]}$ The microscopic control of macroscopic physical properties is an undoubtful benefit to both scientific and technological innovations.

Functional fluorescent molecules have emerged as remarkable materials for advanced applications in bioimaging, ${ }^{[8]}$ photodynamic therapy (PDT), ${ }^{[9]}$ chemo-/biosensing, ${ }^{[10]}$ and optoelectronics. ${ }^{[11]}$ Over the past decades, different physical and chemical properties have 
been utilized to develop smart fluorescent materials that can sense and fastly respond to different external stimulus. ${ }^{[12]}$ Conventional fluorescent molecules are highly emissive in the mono-dispersed state, however their emission is quenched upon aggregate formation. This phenomenon is known as aggregation-caused quenching (ACQ). ${ }^{[13]}$ As the consequence of ACQ effect, the applications of these conventional fluorophores are severely limited. ${ }^{[14]}$ An opposite phenomenon in which the aggregates display brighter luminescence than that single molecule, known as aggregation-induced emission (AIE) was observed and investigated by our group. ${ }^{[15]}$ In past decades, AIE has drawn intensive research focus because it has offered excellent opportunities to explore the unseen meso world and understudied research area of aggregate science. ${ }^{[16]}$ Most of the AIE luminogens (AIEgens) exhibit bright emission in their aggregate state, which can be easily achieved by adding poor solvents to their dilute solutions. ${ }^{[15 b]}$ These aggregates or suspensions formation is an isotropic control without spatial and temporal selectivity and proceeds in a contact way. Therefore if we can develop a spatio-temporal approach in a non-contact way for the aggregation formation, it will have significant technological innovation and application prospects.

Fluorescent soft gels are an essential type of functional optical materials with extensive use in high-tech fields such as information storage, ${ }^{[17]}$ encryption, ${ }^{[18]}$ anti-counterfeiting, ${ }^{[19]}$ luminescent actuators, ${ }^{[20]}$ and wearable optoelectronics. ${ }^{[21]}$ Fluorescent gels with soft pattern systems have promising prospects for their superior applications in the display and readout of encoded information. ${ }^{[22]}$ However, the construction of the soft patterns is exceptionally challenging because the complicated chemical reactions and fabrication techniques. In addition, subtle masks are usually needed to conduct fine patterns, which is extraordinarily laborious and costly. ${ }^{[23]}$ Besides, the encoded information stored in these patterns is static, causing limited commercial application potential. ${ }^{[24]}$ Therefore, using facile stimuli methods such as light to control and directly conduct fluorescent patterns in a direct and non-contact way is highly required. ${ }^{[25]}$ It is anticipated that the current technique can lead to achieving 
precise fabrication of dynamic patterns without masks and can have great commercial application potentials. ${ }^{[26]}$ Recently, AIE-active fluorescent gels prepared by loading AIEgens with common organic gels have been attracting considerable attention. ${ }^{[27]}$ AIEgens emit intensive luminescence in the aggregate state because of the restriction of intramolecular motion (RIM). This feature is inherently compatible with the semi-solid-state characters of gels. ${ }^{[15 b, 22 a]}$ Furthermore, the dispersing medium in gel networks can provide space for AIEgens to motion, so we can regulate their luminescence properties through manipulating the intramolecular motions. ${ }^{[28]}$ Therefore, AIE-active gels exhibit many distinctive functions and show a bright prospect in the smart soft fluorescent pattern construction.

Herein we designed a tetraphenylethene (TPE)-based salicylaldehyde benzoylhydrazone multi-armed AIEgen (TPE-4SAH) that can coordinate with various metal ions. The metal coordinated TPE-4SAH system was used to demonstrate the remote spatio-temporal control of the fluorescence behavior. The emission of the system can only be triggered by UV irradiation and metal ions, while the emission color can be tuned by changing different metal ions such as $\mathrm{Al}^{3+}, \mathrm{Zn}^{2+}$, and $\mathrm{Cd}^{2+}$. Furthermore, the AIEgen as a cross-linker was introduced into a polymeric gel. The formed AIE-active metal coordinated system can realize lasermediated mask-free writing by using a UV laser pen. By further utilizing the time-dependent self-healing property of the hydrazone-based covalent dynamic bonds, dynamic 4D soft patterns with time-gated fluorescence change were successfully achieved, along with the disassembling and reassembling into different patterns with desired dimensionalities. In this research, we have successfully demonstrated an excellent security application by creating digital number-mimicking soft photochromic materials. The strategy of remote spatiotemporal control of material behaviors provides a new way to construct controllable photochromic soft materials with bright application prospects in encryption, information storage, and fine lithography fields.

\section{Results and Discussion}


TPE-4SAH was synthesized by treating an ethanolic solution of 4-methoxybenzhydrazide (AH) with TPE-cored-salicylaldehyde (TPE-4SA). ${ }^{[29]}$ The synthetic route to TPE-4SAH was shown in Figure 1a. The ${ }^{1} \mathrm{H}$ NMR, ${ }^{13} \mathrm{C}$ NMR, and matrix-assisted laser desorption/ionization time-of-flight (MALDI-TOF) mass spectrometry were used to characterize the obtained products, and the corresponding spectra were depicted in Figure S4-S6. TPE-4SAH can respond to different metal ions, and the photoluminescence (PL) spectra of TPE-4SAH in the presence of various metal ions after adequate UV irradiation were recorded (Figure S7). The results revealed that TPE-4SAH could recognize $\mathrm{Al}^{3+}, \mathrm{Cd}^{2+}, \mathrm{Zn}^{2+}$, and $\mathrm{Cd}^{2+}$ with evidential turn-on fluorescence. However, the fluorescence was either quenched or shown insignificant enhancement with the addition of other ions $\left(\mathrm{Li}^{+}, \mathrm{Na}^{+}, \mathrm{K}^{+}, \mathrm{Mg}^{2+}, \mathrm{Ca}^{2+}, \mathrm{Sr}^{2+}, \mathrm{Ce}^{3+}, \mathrm{Cr}^{3+}, \mathrm{Mn}^{2+}\right.$, $\left.\mathrm{Fe}^{3+}, \mathrm{Cu}^{2+}\right)$. The fluorescence responses of $\mathrm{Al}^{3+}$ and $\mathrm{Ga}^{3+}$ ions were similar because they are in the same group of the periodic table.

TPE-4SAH emitted weak orange fluorescence in dimethylformamide (DMF) solution as shown in Figure 1b. The weakly emissive nature of TPE-4SAH can be attributed to different processes such as active intramolecular motions, photo-induced electron transfer (PET), ${ }^{[30]}$ and excited-state intramolecular proton transfer (ESIPT) processes. ${ }^{[31]}$ Notably, common salicylaldehyde benzoylhydrazone systems usually show fluorescence turn-on or ratiometric fluorescence changes after the addition of different metal ions such as $\mathrm{Zn}^{2+}$ and $\mathrm{Al}^{3+}$ into the solutions of probe molecules without additional stimuli. ${ }^{[32]}$ However, the current system TPE4SAH behaves differently. Only the addition of different metal ions to the solution of TPE4SAH resulted in no obvious change in the PL spectra but a drastic fluorescence variation was observed after applying additional stimulus of UV irradiation to the same solvent mixtures $\left(\mathrm{TPE}-4 \mathrm{SAH}+\mathrm{M}^{\mathrm{n}+}\right)$. 
a

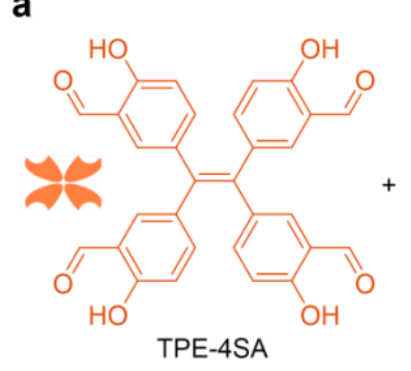

b

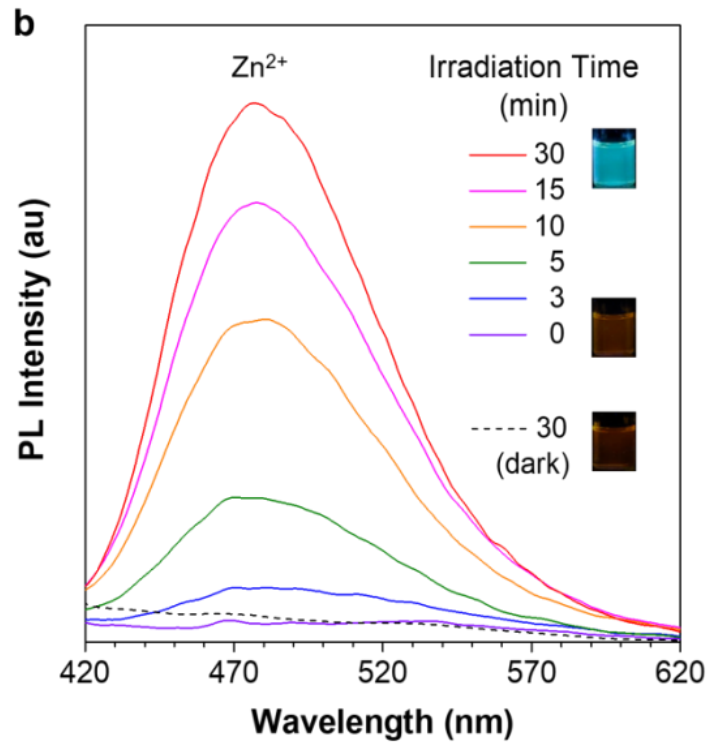

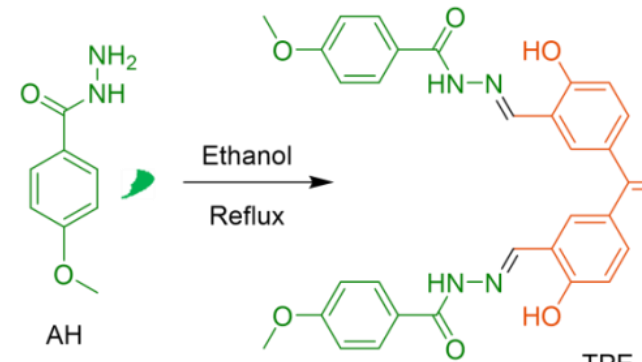

TPE-4SAH
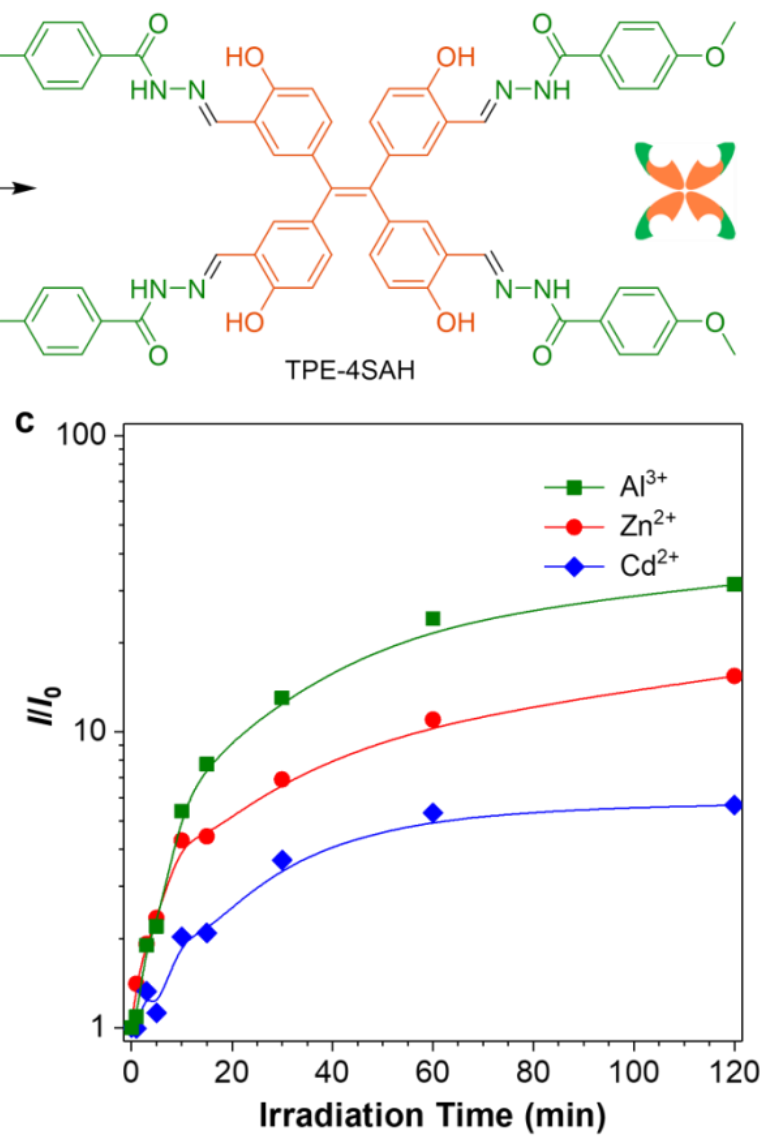

Figure 1. (a) Synthetic route to TPE-4SAH, a TPE-based AIEgen with four arms that can coordinate with different metal ions. (b) Time dependent photoluminescence (PL) spectra of TPE-4SAH $(10 \mu \mathrm{M})$ in $\mathrm{DMF} / \mathrm{H}_{2} \mathrm{O}(8: 2, \mathrm{v} / \mathrm{v})$ mixtures in the presence of $\mathrm{Zn}^{2+}(0.5 \mathrm{mM})$ measured after UV irradiation. Insets: fluorescent images of non-radiated and radiated samples after addition of $\mathrm{Zn}^{2+}$ ions. (c) Time dependent PL intensity changes for TPE-4SAH $(10 \mu \mathrm{M})$ in $\mathrm{DMF} / \mathrm{H}_{2} \mathrm{O}(8: 2, \mathrm{v} / \mathrm{v})$ mixtures in the presence of $\mathrm{Al}^{3+}, \mathrm{Zn}^{2+}$, and $\mathrm{Cd}^{2+}$ ions $(c=0.5$ $\mathrm{mM}$ ), where $I=$ maximum PL intensity at a specific irradiation time and $I_{0}=$ maximum PL intensity at irradiation time of $0 \mathrm{~min}$.

Take the TPE-4SAH/ $/ \mathrm{Zn}^{2+}$ system as a model system. As shown in Figure $1 \mathrm{~b}$, the addition of the aqueous solution of $\mathrm{Zn}^{2+}$ to the DMF solution of TPE-4SAH (DMF/H $\left.\mathrm{H}_{2} \mathrm{O}=8: 2, \mathrm{v} / \mathrm{v}\right)$ exhibited no obvious change in the fluorescence. However, after applying the $365 \mathrm{~nm}$ UV light, the fluorescence was significantly intensified and showed a hypsochromic shift with an increasing irradiation time. Similar results were obtained in the case of TPE-4SAH/Al ${ }^{3+}$ and TPE-4SAH/Cd ${ }^{2+}$ (Figure S9-S10). The time-dependent PL intensity changes for TPE-4SAH in the presence of $\mathrm{Al}^{3+}, \mathrm{Zn}^{2+}$, and $\mathrm{Cd}^{2+}$ after the $\mathrm{UV}$ irradiation were summarized and found to have different PL responses in the presence of different metal ions after UV irradiation. For 
example, TPE-4SAH/A1 ${ }^{3+}$ system showed 31-fold PL enhancement after $120 \mathrm{~min}$ of UV irradiation. Similarly, TPE-4SAH/ $/ \mathrm{Zn}^{2+}$ and TPE-4SAH/Cd ${ }^{2+}$ systems showed 15 -fold and 6fold PL enhancement respectively after $120 \mathrm{~min}$ of UV irradiation (Figure 1c). These results indicate that different metal ions have different binding abilities with the probe molecule and that could be responsible for the time-dependent PL intensities change upon the UV irradiation. More importantly, TPE-4SAH showed time-dependent emission wavelength change after the addition of different metal ions under UV irradiation. The fluorescence emission of the TPE-4SAH/Al ${ }^{3+}$ system showed an obvious hypsochromic shift of $115 \mathrm{~nm}$ compared to the TPE-4SAH/Zn ${ }^{2+}$ and TPE-4SAH/Cd ${ }^{2+}$ systems.

This unique UV irradiation-mediated fluorescence change behavior of the above TPE4SAH/M $\mathrm{M}^{\mathrm{n}+}$ systems was further explored with the help of ${ }^{1} \mathrm{H}$ NMR and MALDI-TOF mass measurements. The ${ }^{1} \mathrm{H}$ NMR spectra of TPE-4SAH/ $\mathrm{Zn}^{2+}$ where the signal of proton from the $\mathrm{N}-\mathrm{NH}$ - group which was first observed at $12.2 \mathrm{ppm}$ shifted to the low field in the both spectra collected with and without UV irradiation (Figure 2a). The signal peak at $12.2 \mathrm{ppm}$ also became very broad, but the integral remained unchanged. Meanwhile, the signal of proton from the phenolic hydroxyl group (-OH) at around $11.6 \mathrm{ppm}$ disappeared in the conditions of with and without UV irradiation, which indicates that coordination between TPE-4SAH and the metal ion occurred, and the hydroxyl group participated in the coordination process regardless of UV irradiation was confirmed. UV-vis spectra of the TPE-4SAH/Zn ${ }^{2+}$ system with and without the UV irradiation were measured and no obvious difference was found (Figure S8). In addition, the MALDI-TOF mass spectra of TPE-4SAH with $\mathrm{Zn}^{2+}$ before (Figure S11) and after UV irradiation (Figure S12) was also determined. The mass spectra showed four peaks at $\mathrm{m} / \mathrm{z}=1163.2233,1227.1231,1291.0233,1353.9218$ and which can be attributed to the fragments $[(\mathrm{TPE}-4 \mathrm{SAH})+\mathrm{Zn}]^{+},[(\mathrm{TPE}-4 \mathrm{SAH})+2 \mathrm{Zn}+\mathrm{H}]^{+},[(\mathrm{TPE}-4 \mathrm{SAH})+$ $3 \mathrm{Zn}+2 \mathrm{H}]^{+}$, and $[(\mathrm{TPE}-4 \mathrm{SAH})+4 \mathrm{Zn}]^{+}$respectively, confirming the formation of four coordinated TPE-4SAH/Zn ${ }^{2+}$ complex system. These results reveal that coordination between 
TPE-4SAH and the metal ion occurs regardless of the UV irradiation. In addition, no photoreactions are involved in TPE-4SAH/metal ion complex under UV irradiation.
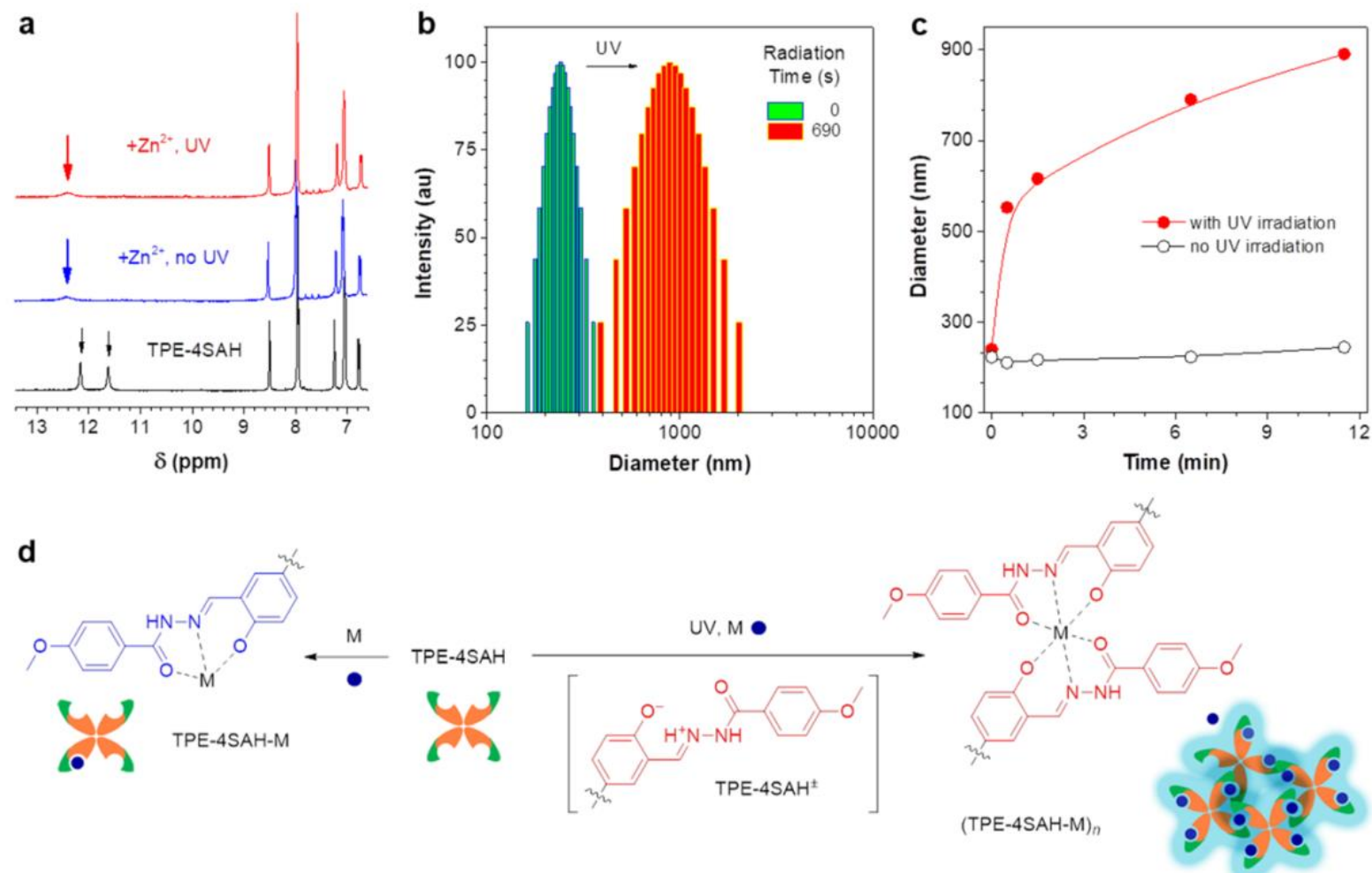

Figure 2. (a) ${ }^{1} \mathrm{H}$ NMR spectra $\left(\mathrm{DMF}-d_{7}-\mathrm{H}_{2} \mathrm{O} ; 8: 2\right.$, v/v) of TPE-4SAH and TPE-4SAH $+\mathrm{Zn}^{2+}$ before and after UV irradiation; [TPE-4SAH] $=3 \mathrm{mM},\left[\mathrm{Zn}^{2+}\right]=0.6 \mathrm{mM}$. (b) DLS profiles of TPE-4SAH $(10 \mu \mathrm{M})$ with $\mathrm{Zn}^{2+}(0.5 \mathrm{mM})$ in a $\mathrm{DMF} / \mathrm{H}_{2} \mathrm{O}$ mixture $(8: 2, \mathrm{v} / \mathrm{v})$ upon UV irradiation for $0 \mathrm{~s}$ and $690 \mathrm{~s}$. (c) Plots of particle size against UV irradiation time for TPE4SAH $(10 \mu \mathrm{M})$ with $\mathrm{Zn}^{2+}(0.5 \mathrm{mM})$ in the $\mathrm{DMF} / \mathrm{H}_{2} \mathrm{O}$ mixture $(8: 2, \mathrm{v} / \mathrm{v})$ with and without UV irradiation. (d) Proposed mechanism of photo-triggered aggregation-induced emission (PTAIE) process.

Furthermore, the size of the complexes based on the TPE- $4 \mathrm{SAH} / \mathrm{Zn}^{2+}$ system was determined by using dynamic light scattering (DLS) measurement. TPE-4SAH was first dissolved in DMF, and $\mathrm{Zn}^{2+}$ aqueous solution was added (Figure 2b). The average size of the complex before applying UV irradiation was measured and found to be $\sim 240 \mathrm{~nm}$. However, after applying the UV light, the size of the complex increased dramatically in the first $30 \mathrm{~s}$ and then gradually increased up to $\sim 900 \mathrm{~nm}$ in $11.5 \mathrm{~min}$ (Figure $2 \mathrm{c}$ ). In contrast, the aggregate size of the sample without UV irradiation displayed no obvious increase. The size of TPE-4SAH aggregates in $\mathrm{DMF} / \mathrm{H}_{2} \mathrm{O}$ mixture without $\mathrm{Zn}^{2+}$ was also measured, and no obvious difference in the aggregate diameter with and without UV irradiation was found 
(Figure S13). These results indicate that UV irradiation could induce the aggregate formation after mixing the TPE-4SAH and the metal ion in the DMF/water mixture.

Density functional theory (DFT)-based calculations were conducted to further explore the binding mechanism between the AIEgen and different metal ions. Salicylaldehyde methoxybenzoylhyrazone (SA-BH) was used as the model compound to mimic the arm of TPE-4SAH. The Gibbs free energy change $\left(\Delta \mathrm{G}^{\circ}\right)$ of the complex between SA-BH and $\mathrm{Zn}^{2+}$ was calculated by ORCA 4.2.1. As shown in Figure S17, $\Delta \mathrm{G}^{\circ}$ of the complex in the excited state energy ($\left.17.19 \mathrm{kcal} \mathrm{mol}^{-1}\right)$ is lower than that in the ground state energy $\left(-16.57 \mathrm{kcal} \mathrm{mol}^{-1}\right)$, which indicates that $\mathrm{SA}-\mathrm{BH}$ in the excited state has a stronger binding affinity with $\mathrm{Zn}^{2+}$. In the excited state, the charge separation process of SA-BH is much easier to occur, and this leads to tight coordination between the binding sites and the metal ions.

Based on the above results, it is speculated that the four-armed AIEgen TPE-4SAH-based system after the addition of the metal ions are relative dispersive in solution and only form loose coordination complex. We believed that most metal ions do not participate in the coordination or coordinate with a single ligand (salicylaldehyde methoxy-benzoylhyrazone part) due to diffusion and viscosity limitation. The active intramolecular motions of this AIEgen-based complex lead to weak fluorescence. After applying the UV light, the charge separation process is much easier to occur for the TPE-4SAH in the excited state, which leads to a stronger binding affinity with the metal ion. The large interaction force improves the ability to overcome the diffusion and accelerates the tighter and bigger aggregates. The UV irradiation also triggers the molecular motion and benefits for generating intermolecular interactions, ${ }^{[33]}$ which may increase the possibility for the four-armed TPE molecules to generate intermolecular/ intramolecular coordination with the metal ion via the metal-bridged crosslinking/coordination-induced complexation mechanism. ${ }^{[32]}$ The PL intensity enhancement after the formation of bigger aggregates can be attributed to the restriction of intramolecular motions (RIM) via intermolecular coordination. This unusual phenomenon of 
photo-induced aggregate formation with enhanced PL intensity was coined as photo-triggered aggregation-induced emission (PTAIE) (Figure 2d). Meanwhile, the blue-shifted emission of the molecule could be caused by the weakened ESIPT process. Based on the calculation results by ORCA 4.2.1, the excited state complex formation for (TPE-4SAH-M) $n$ is found to be more feasible compared to its ground state because the free energy of excited sate was found to be $117 \mathrm{kcal} \mathrm{mol}^{-1}$ which is lower than its ground state free energy. The different PL intensity changes after binding with different metal ions such as $\mathrm{Al}^{3+}, \mathrm{Zn}^{2+}$, and $\mathrm{Cd}^{2+}$ were also explored with the help of their binding constant measurement and complexation energy calculation results (Table S1 and Figure S16). These results further support the different fluorescence response rates and emission wavelengths of the respective system after treating TPE-4SAH with different metal ions $\left(\mathrm{Al}^{3+}, \mathrm{Zn}^{2+}\right.$, and $\left.\mathrm{Cd}^{2+}\right)$ in the presence and absence of the UV irradiation.

Based on the above discovery and investigation results, TPE-4SAH/metal ion-based PTAIE system was integrated into polymeric networks to fabricate fluorescent gels in order to showcase the strategy to realize the microscopic control of macroscopic behaviors in a spatiotemporal manner and apply the gels in advanced optical fields. Linear acylhydrazineterminated PEG (PEG-2AH) was synthesized according to the method in our previous work. $^{[34]}$ Utilizing the reaction between aldehyde and hydrazine groups, an AIE-active polymeric gel (PTPEG) was prepared in DMF with PEG-2AH and TPE-4SA as the network chain and cross-linker, respectively (Figure 3a). The synthesized PEG-2AH, TPE-4SA, and PTPEG xerogel were characterized ATR-FTIR measurement. The newly appeared band around $1650 \mathrm{~cm}^{-1}$ which is assigned to the $\mathrm{C}=\mathrm{N}$ group demonstrates the formation of acylhydrazone group in the gel network and confirmed the inclusion of TPE-4SAH part into the polymeric gel network (Figure S14). 


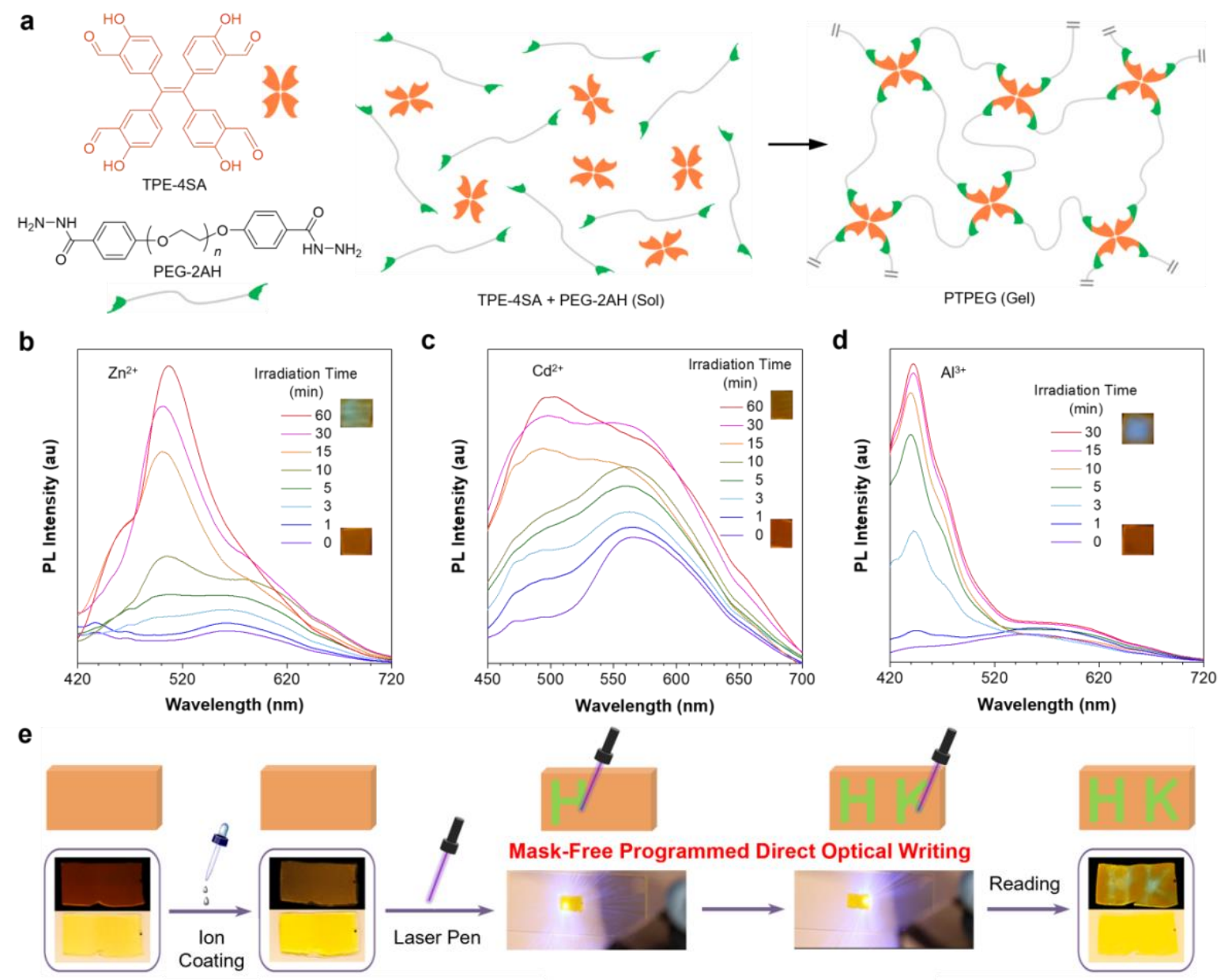

Figure 3. (a) Schematic illustration for the preparation of PTPEG gel. Fluorescence spectra of PTPEG gels coated with (b) $\mathrm{Zn}^{2+}$, (c) $\mathrm{Cd}^{2+}$ and (d) $\mathrm{Al}^{3+}$ aqueous solutions $(1 \mathrm{mM})$ measured at different UV irradiation times. Insets are fluorescent images of the starting and irradiated gels. (e) Demonstration of photopattern generation from the gels through a mask-free programmed direct optical writing process.

After the manipulation of AIE-active gel, the UV-mediated fluorescence change behavior through the PTAIE process of the TPE-4SAH/metal ion system was verified. To support this, the aqueous solutions of different metal ions such as $\mathrm{Zn}^{2+}, \mathrm{Cd}^{2+}$, and $\mathrm{Al}^{3+}$ were coated on the surface of the PTPEG gel. After UV irradiation, a noticeable fluorescence enhancement was observed on the PTPEG gels which can be attributed to the photo-triggered aggregationinduced emission (PTAIE) mechanism. The time-dependent UV exposure to the $\mathrm{Zn}^{2+}$ coated gels was studied where a hypsochromic band $\left(\lambda_{\max } \approx 510 \mathrm{~nm}\right)$ with enhanced PL intensity was observed with increasing irradiation time. An obvious emission color change can be seen from the inset photographs where the gels coated with $\mathrm{Zn}^{2+}$ turns to be green after $1 \mathrm{~h}$ of UV 
irradiation (Figure 3b). Similarly, the gels coated with $\mathrm{Cd}^{2+}$ (Figure 3c) and $\mathrm{Al}^{3+}$ (Figure 3d) turn to be yellow and blue, respectively under UV irradiation. These results well matched with the photo-triggered AIE process in the solution state, as illustrated in Figure 1. This indicates that the PTAIE-induced fluorescence change is also effective in the gel-based system benefiting from the gel character of the existence of both condensed phase and free space. Because the network structure of the gel has blocking effect to the ions in terms of ionic radius, the $\mathrm{Cd}^{2+}$ complexation with TPE-4SAH may be affected more. This effect distinguishes the $\mathrm{Cd}^{2+}$-based system from the other two systems and make the three systems show obvious different emission colors.

Remote manipulation of luminescence behaviors of materials has been pursued for advanced optical applications in the field of information technology. Inspired by the result of UV-mediated fluorescence change behavior of the PTPEG/ $/ \mathrm{Z}^{2+}$ system, remote encoding encrypted information and readout were realized. Figure 3e shows the direct writing of information on the surface of $\mathrm{Zn}^{2+}$ coated PTPEG gel using a UV laser pen. During the writing process, the laser-exposed regions turned to green fluorescence, while the unexposed areas remained the original orange fluorescence. Using this method, characters " $\mathrm{H}$ " and " $\mathrm{K}$ " as the information was successfully encoded on the soft matrix. Because the information is fluorescent, it can be only encrypted under UV light. Compared with the traditional maskmediated information encoding method, this mask-free manner is much more convenient and economical. This method also holds great significance in the field of photolithography and other innovative areas.

Materials with acylhydrazone dynamic covalent bonds have been widely reported to have self-healing property, ${ }^{[35]}$ and our previous report revealed that this self-healing property is also time dependent. ${ }^{[36]}$ We found that this time-dependent self-healing property was also applicable to the PTPEG gel. As demonstrated in Figure $\mathbf{4 b}$ and $\mathbf{4 c}$, the self-healing efficiency was changed over various self-healing time. As the healing time increases, the 
healing efficiency is higher. This means that we can modulate the degree of self-healing by adjusting the healing time.

a
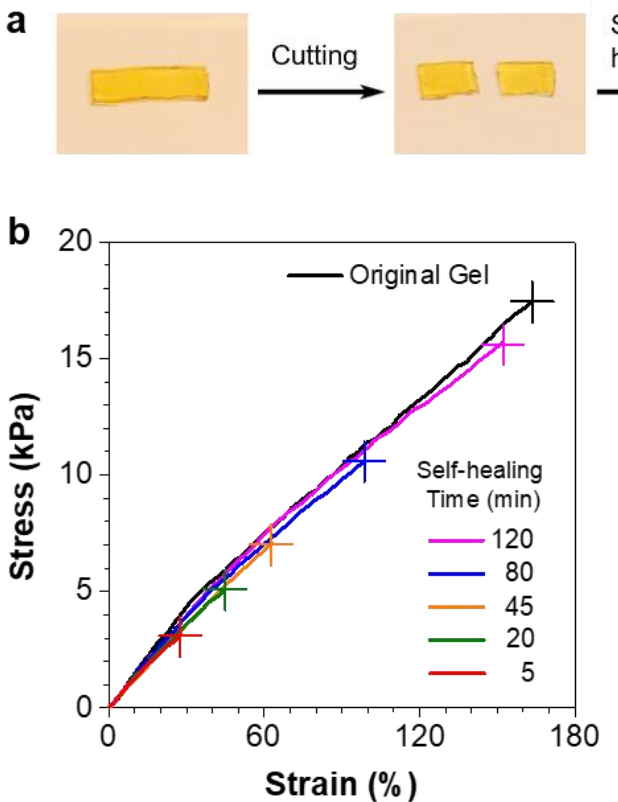
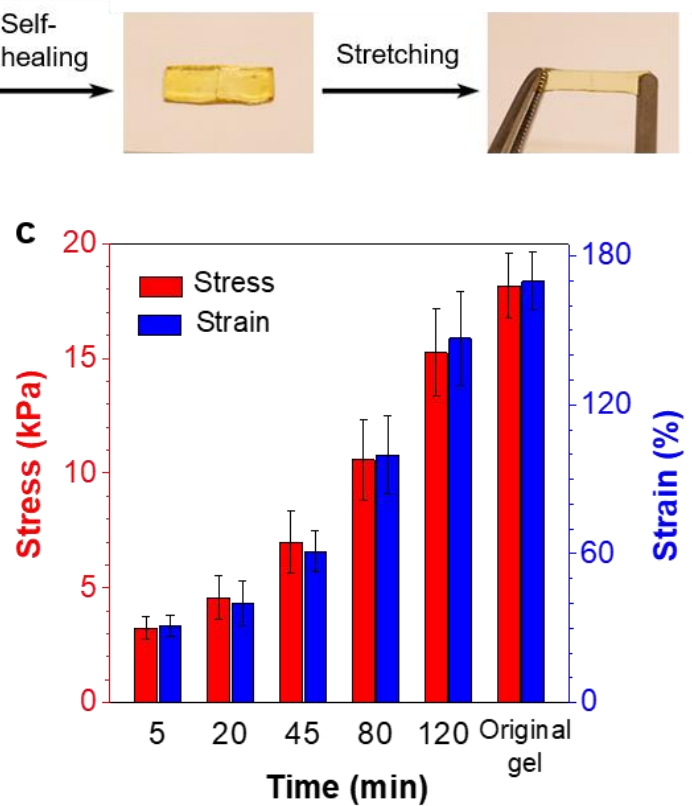

Figure 4. (a) Photographs showing self-healing property of PTPEG gel. (b) Stress-strain plots of the starting material (original gel) and the gels after self-healed for different periods. (c) Time dependence of mechanical properties of the self-healed gels.

Based on the time-dependent PTAIE behavior and time-dependent self-healing property of the PTPEG/metal ion system. Transformable four-dimensional (4D) soft patterns were conducted to demonstrate the microscopic control of macroscopic behaviors of a material through the spatio-temporal manner. As illustrated in Figure 5 and Movie 1, nine flaky PTPEG gels with identical sizes were prepared followed by coating aqueous solutions of $\mathrm{Al}^{3+}$, $\mathrm{Zn}^{2+}$, and $\mathrm{Cd}^{2+}$, respectively, on different flakes. Then the flakes were assembled and healed into a $3 \times 3$ two-dimensional (2D) array which could be picked up by tweezers. The array was soft because its matrix was polymeric gel. The array did not show fluorescent patterns without UV irradiation, so it could be regarded as an encrypted pattern. After applying the decoding key of UV light, chromic fluorescence of $\mathrm{Al}^{3+}$ coated and $\mathrm{Zn}^{2+}$ coated flakes gradually appeared because of their rapid response. The light-colored, multi-color fluorescent pattern constitutes a three-dimensional (3D) pattern I. Because the fluorescence changes of PTPEG/metal ion systems based on $\mathrm{Cd}^{2+}, \mathrm{Zn}^{2+}$, and $\mathrm{Al}^{3+}$ have different responsive rates to 
UV irradiation and the emission intensity for the specific system also exhibited irradiation time dependency, the 3D pattern I changed with the extension of the irradiation time. The emission intensity of $\mathrm{Zn}^{2+}$ and $\mathrm{Al}^{3+}$ coated flakes increased significantly and chromic fluorescence of $\mathrm{Cd}^{2+}$ coated flakes began to occur. This array with obvious different multicolor constituted 3D pattern II. With further extension of the irradiation time, emission intensity of $\mathrm{Cd}^{2+}$ coated flakes increased, and 3D pattern III formed. The evolution from 3D pattern I to $3 \mathrm{D}$ pattern II to $3 \mathrm{D}$ pattern III along the timeline constituted the time-gated $4 \mathrm{D}$ patterning. Moreover, the healing efficiency of PTPEG gels did not reach the high level within the timescale, the healed gels array could be disassembled. Thus, after repatterning the disassembled gels and healing again, soft 3D pattern IV could be achieved. Utilizing the timedependent self-healing property, soft 2D patterns could be also constructed with the linear multicolour pattern. The 4D soft pattern largely expands the diversity of patterns, which revealed bright potential be used as wearable optoelectronic devices.

The PTPEG/ $/ \mathrm{Zn}^{2+}$ photochromic system could also act as security display materials with error correction ability. We assumed that letters "A", "I", and "E" in the sequence of "AIE" were correct and other sequences were wrong. As shown in Figure S18, three flaky PTPEG gels with identical sizes were prepared and written with letters "I", "A", and "E", respectively, with $\mathrm{Zn}^{2+}$ aqueous solution. Then the flakes were assembled through short time healing. The letters could not be recognized either under day-light or UV light with a high security level. After applying adequate UV irradiation, the letters appeared green fluorescence with the wrong order. The flakes could be disassembled and rearranged. After healing again, the corrected information was displayed. 


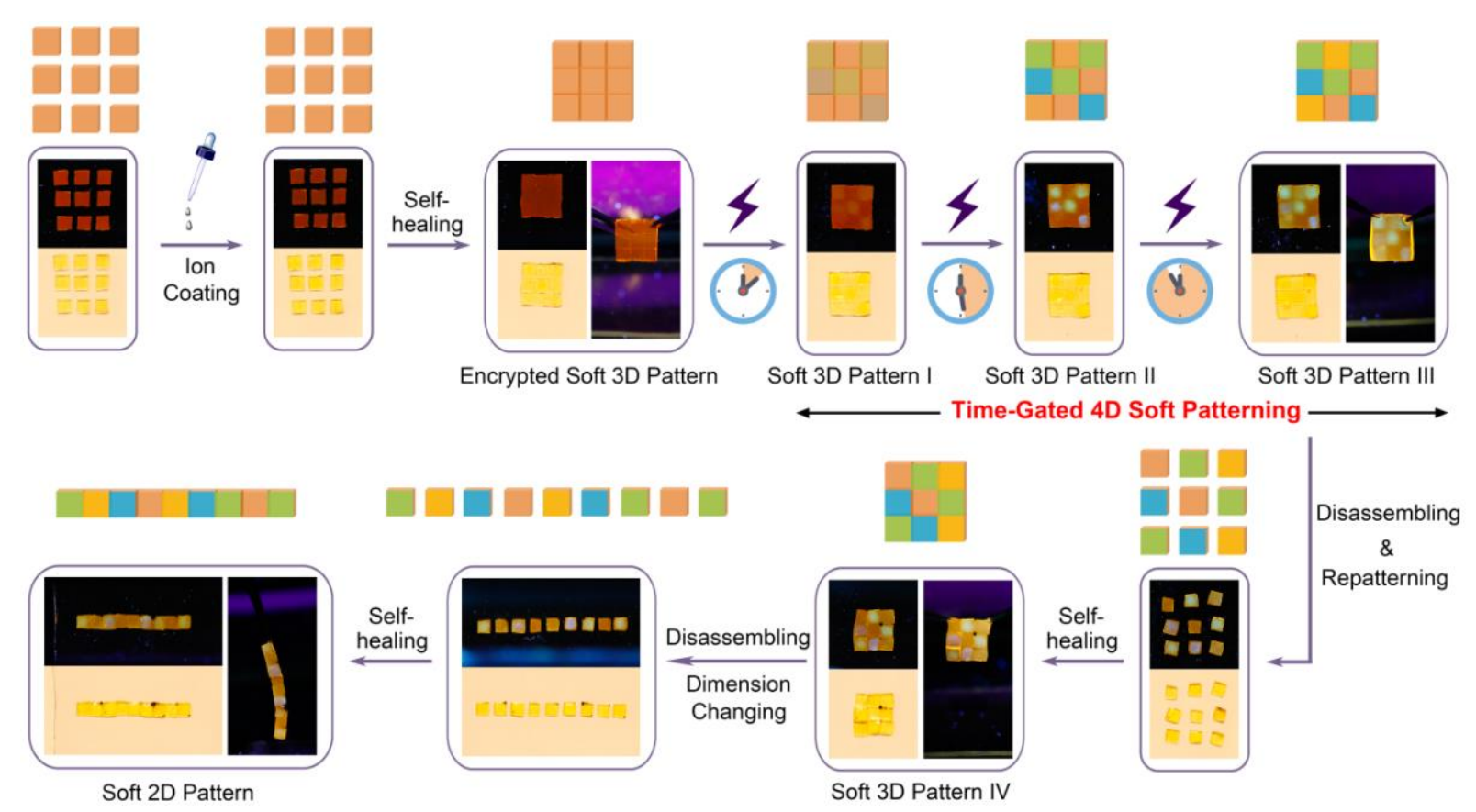

Figure 5. Demonstration of photopattern generation from PTPEG gels: coating the gel with ions, formation of a 3D pattern via self-healing of the ion-coated gels, generation of 4D soft patterns with time-gated color change, disassembling and reassembling into patterns with desired dimensionalities.

In the PTPEG/ $\mathrm{Zn}^{2+}$ and PTPEG/Al ${ }^{3+}$ systems, encrypted transformable soft digital information displaying materials could also be achieved. 14 PTPEG strips were prepared and arranged to digitals " 88 " as shown in Figure S19. After short time healing, two soft digital displaying frameworks formed. Coating $\mathrm{Zn}^{2+}$ aqueous solution on different strips on the left framework followed by adequate UV irradiation afforded green fluorescent digital “2”. Blue fluorescent digital "9" was also prepared in the similar way by using $\mathrm{Al}^{3+}$ solution. After disassembling the two "digitals", rearranging the position, and re-healing the strips, new digitals information " 35 " could be attained.

\section{Conclusion}

In summary, a strategy that realizes remote spatio-temporal control of luminescence behavior of materials through photo-triggered aggregation-induced emission (PTAIE) process is reported. TPE-based salicylaldehyde benzoylhydrazone four-arm AIEgen, TPE-4SAH has been designed and synthesized. It was found that the TPE-4SAH/metal ion system shows fluorescence changes under the trigger of UV irradiation and the time. The photochromic 
process was also gated by the ion species $\left(\mathrm{Al}^{3+}, \mathrm{Zn}^{2+}\right.$, and $\left.\mathrm{Cd}^{2+}\right)$. The mechanism of the above fluorescence behaviors was investigated and the PTAIE process was summarized to be responsible for them. Furthermore, by integrating the TPE-4SAH part as the cross-linking point in a PEG-based polymeric network, an self-healable soft AIE-active gel, PTPEG has been fabricated for which PTAIE-mediated fluorescence change behaviors also effect with the addition of the metal ions. Based on the PTPEG/metal ion system, a strategy of remote spatiotemporal control of material behaviors was demonstrated by utilizing time-dependent PTAIE process and self-healing property. A non-contact mask-free writing process and dynamic soft 4D patterns were achieved. Security display soft materials and photochromic transformable soft digital materials were also fabricated. Overall, the new route of microscopic control of macroscopic behaviors of materials reported here is promising for development of advanced smart materials and manipulation of them. The gel system constructed here has bright prospects in optoelectronic applications in high-tech fields.

\section{Acknowledgements}

The authors acknowledge funding from the National Natural Science Foundation of China (21788102 and 22005028), the Research Grants Council of Hong Kong (N-HKUST609/19, 1606620 and C6009-17G), the Innovation and Technology Commission (ITCCNERC14SC01), the National Key Research and Development Program of China (2018YFE0190200), the Science and Technology Plan of Shenzhen (JCYJ20200109110608167，JCYJ20180507183832744，JCYJ20180306180231853), Beijing Institute of Technology Research Fund Program for Young Scholars (XSQD-202023002).

\section{Conflict of Interest}

The authors declare no conflict of interest.

Received: ((will be filled in by the editorial staff))

Revised: ((will be filled in by the editorial staff)) Published online: ((will be filled in by the editorial staff)) 
References

[1] D. L. Goodstein, States of Matter. Dover, New York, 1975.

[2] P. Papon, J. Leblond, P. H. E. Meijer, Thermodynamics and Statistical Mechanics of Phase Transitions. In: The Physics of Phase Transitions. Advanced Texts in Physics, Springer, Berlin, Heidelberg, 2002.

[3] G. Jaeger, Arch. Hist. Exact Sc. 1998, 53, 51.

[4] V. V. Kocharovsky, V. V. Kocharovsky, Phys. Lett. A 2015, 379, 466.

[5] a) P. Zalden, F. Quirin, M. Schumacher, J. Siegel, S. Wei, A. Koc, M. Nicoul, M. Trigo, P. Andreasson, H. Enquist, M. J. Shu, T. Pardini, M. Chollet, D. Zhu, H. Lemke, I. Ronneberger, J. Larsson, A. M. Lindenberg, H. E. Fischer, S. Hau-Riege, D. A. Reis, R. Mazzarello, M. Wuttig, K. Sokolowski-Tinten, Science 2019, 364, 1062; b) B. Kang, Y. Lee, T. Piao, Z. Ding, W. D. Wang, Mater. Horiz. 2021, 8, 939; c) Y. Li, H Yan, B. Xu, L. Zhen, C.-Y. Xu, Adv. Mater. 2021, 33, 2000581.

[6] a) W. M. Winslow, U.S. Patent 2, 1947; b) W. M. Winslow, J. Appl. Phys. 1949, 20, 1137.

[7] T. C. Halsey, Science 1992, 258, 761.

[8] a) M. Schäferling, Angew. Chem. Int. Ed. 2012, 51: 3532; b) Z. Sheng, B. Guo, D. Hu, S. Xu, W. Wu, W. H. Liew, K. Yao, J. Jiang, C. Liu, H. Zheng, B. Liu, Adv. Mater. 2018, 30, 1800766; c) J. V. Frangioni, Curr. Opin. Chem. Biol. 2003, 7, 626; d) X. Qian, Z. Xu, Chem. Soc. Rev. 2015, 44, 4487.

[9] a) S. S. Lucky, K. C. Soo, Y. Zhang, Chem. Rev. 2015, 115, 1990; b) S. Kwiatkowski, B. Knap, D. Przystupski, J. Saczko, E. Kędzierska, K. Knap-Czop, J. Kotlińska, O. Michel, K. Kotowski, J. Kulbacka, Biomed. Pharmacother. 2018, 106, 1098; c) J. Chen, T. Fan, Z. Xie, Q. Zeng, P. Xue, T. Zheng, Y. Chen, X. Luo, H. Zhang, Biomaterials 2020, 237, 119827.

[10] a) K. Siddharth, P. Alam, M. D. Hossain, N. Xie, J. S. Nambafu, F. Rehman, J. W. Y. Lam, G. Chen, J. Cheng, Z. Luo, G. Chen, B. Z. Tang, M. Shao, J. Am. Chem. Soc. 2021, 143, 
2433; b) H. Xie, J. Zhang, C. Chen, F. Sun, H. Liu, X. He, K. W. K. Lam, Z. Li, J. W. Y. Lam, G.-Q. Zhang, D. Ding, R. T. K. Kwok, B. Z. Tang, Mater. Chem. Front. 2021, 5, 1830; c) J. S. Marvin, Y. Shimoda, V. Magloire, M. Leite, T. Kawashima, T. P. Jensen, I. Kolb, E. L. Knott, O. Novak, K. Podgorski, N. J. Leidenheimer, D. A. Rusakov, M. B. Ahrens, D. M. Kullmann, L. L. Looger, Nat. Methods 2019, 16, 763.

[11] a) T. Higuchi, H. Nakanotani, C. Adachi, Adv. Mater. 2015, 27, 2019; b) A. Salehi, C. Dong, D.-H. Shin, L. Zhu, C. Papa, A. T. Bui, F. N. Castellano, F. So, Nat. Commun. 2019, $10,2305$.

[12] a) R. Kumar, A. Sharma, H. Singh, P. Suating, H. S. Kim, K. Sunwoo, I. Shim, B. C. Gibb, J. S. Kim, Chem. Rev. 2019, 119, 9657; b) S. Nocentini, D. Martella, C. Parmeggiani, S. Zanotto, D. S. Wiersma, Adv. Opt. Mater. 2018, 6, 1800167; c) Z. Li, Y. Yin, Adv. Mater. 2019, 31, 1807061.

[13] W. Z. Yuan, P. Lu, S. Chen, J. W. Y. Lam, Z. Wang, Y. Liu, H. S. Kwok, Y. Ma, B. Z. Tang, Adv. Mater. 2010, 22, 2159.

[14] Y. Hong, J. W. Y. Lam, B. Z. Tang, Chem. Soc. Rev. 2011, 40, 5361.

[15] a) J. Luo, Z. Xie, J. W. Y. Lam, L. Cheng, H. Chen, C. Qiu, H. S. Kwok, X. Zhan, Y. Liu, D. Zhu, B. Z. Tang, Chem. Commun. 2001, 1740; b) J. Mei, N. L. C. Leung, R. T. K. Kwok, J. W. Y. Lam, B. Z. Tang, Chem. Rev. 2015, 115, 11718.

[16] a) Z. Zhao, H. Zhang, J. W. Y. Lam, B. Z. Tang, Angew. Chem. Int. Ed. 2020, 59, 9888; b) J. Yang, M. Fang, Z. Li, Aggregate 2020, 1, 6.

[17] H. Wang, X. Ji, Z. A. Page, J. L. Sessler, Mater. Chem. Front. 2020, 4, 1024.

[18] H. Wang, X. Ji, Z. Li, C. N. Zhu, X. Yang, T. Li, Z. L. Wu, F. Huang, Mater. Chem. Front. 2017, 1, 167.

[19] Y. Zhang, X. Le, Y. Jian, W. Lu, J. Zhang, T. Chen, Adv. Funct. Mater. 2019, 29, 1905514. 
[20] S. Wei, W. Lu, X. Le, C. Ma, H. Lin, B. Wu, J. Zhang, P. Theato, T. Chen, Angew. Chem. Int. Ed. 2019, 58, 16243.

[21] a) G.-H. Lee, H. Moon, H. Kim, G. H. Lee, W. Kwon, S. Yoo, D. Myung, S. H. Yun, Z. Bao, S. K. Hahn, Nat. Rev. Mater. 2020, 5, 149; b) A. K. Yetisen, J. L. Martinez-Hurtado, B. Ünal, A. Khademhosseini, H. Butt, Adv. Mater. 2018, 30, 1706910.

[22] a) Z. Li, X Ji,. H. Xie, B. Z. Tang, Adv. Mater. DOI: 10.1002/adma.202100021; b) W. Lu, S. Wei, H. Shi, X. Le, G. Yin, T. Chen, Aggregate 2021, 1.

[23] X. Ji, X. Chi, M. Ahmed, L. Long, J. L. Sessler, Acc. Chem. Res. 2019, 52, 1915.

[24] a) X. Yu, H. Zhang, J. Yu, Aggregate 2021, 2, 20; b) Y. Ma, Y. Yu, P. She, J. Lu, S. Liu, W. Huang, Q. Zhao, Sci. Adv. 2020, 6, eaaz2386.

[25] a) X. Jia, C. Shao, X. Bai, Q. Zhou, B. Wu, L. Wang, B. Yue, H. Zhu, L. Zhu, Proc. Natl. Acad. Sci. U.S.A. 2019, 116, 4816; b) Z. Zhang, L. Cheng, J. Zhao, H. Zhang, X. Zhao, Y. Liu, R. Bai, H. Pan, W. Yu, X. Yan, J. Am. Chem. Soc. 2021, 143, 902; c) A. K. Yetisen, H. Butt, F. da Cruz Vasconcellos, Y. Montelongo, C. A. B. Davidson, J. Blyth, L. Chan, J. B. Carmody, S. Vignolini, U. Steiner, J. J. Baumberg, T. D. Wilkinson, C. R. Lowe, Adv. Opt. Mater. 2014, 2, 250; d) Z. Li, S. Su, L. Yu, Z. Zheng, X. Wang, Soft Matter 2018, 14, 2767. [26] a) X. Ji, R.-T. Wu, L. Long, X.-S. Ke, C. Guo, Y.-J. Ghang, V. M. Lynch, F. Huang, J. L. Sessler, Adv. Mater. 2018, 30, 1705480; b) Q. Wang, Q. Zhang, Q.-W. Zhang, X. Li, C.-X. Zhao, T.-Y. Xu, D.-H. Qu, H. Tian, Nat. Commun. 2020, 11, 158.

[27] a) C.-W. Zhang, B. Ou, S.-T. Jiang, G.-Q. Yin, L. J. Chen, L. Xu, X. Li, H.-B. Yang, Polym. Chem. 2018, 9, 2021; b) S. S. Liow, Q. Dou, D. Kai, Z. Li, S. Sugiarto, C. Y. Y. Yu, R. T. K. Kwok, X. Chen, Y.-L. Wu, S. T. Ong, A. Kizhakeyil, N. K. Verma, B. Z. Tang, X. J. Loh, Small 2017, 13, 1603404; c) Z. Li, P. Liu, X. Ji, J. Gong, Y. Hu, W. Wu, X. Wang, H.-Q. Peng, R. T. K. Kwok, , J. W. Y. Lam, J. Lu, B. Z. Tang, Adv. Mater. 2020, 32, 1906493.

[28] K. Cui, J. P. Gong, Aggregate 2021, 2:e33.

[29] F. Wang, X. Zeng, X. Zhao, H. Lu, Q. Wang, J. Lumin. 2019, 208, 302. 
[30] Y. Cui, D. Yue, Y. Huang, J. Zhang, Z. Wang, D. Yang, G. Qian, Chem. Commun., 2019, 55, 11231.

[31] a) A. C. Sedgwick, L. Wu, H.-H. Han, S. D. Bull, X.-P. He, T. D. James, J. L. Sessler, B. Z. Tang, H. Tian, J. Yoon, Chem. Soc. Rev. 2018, 47, 8842; b) P. Alam, N. L. C. Leung, Y. Cheng, H. Zhang, J. Liu, W. Wu, R. T. K. Kwok, J. W. Y. Lam, H. H. Y. Sung, I. D. Williams, B. Z. Tang, Angew. Chem. Int. Ed. 2019, 58, 4536.

[32] P. Alam, N. L. C. Leung, J. Zhang, R. T. K. Kwok, J. W. Y. Lam, B. Z. Tang, Coord. Chem. Rev. 2021, 429, 213693.

[33] K. Chen, R. Zhang, G. Li, B. Li, Y. Ma, M. Sun, Z. Wang, B. Z. Tang, Mater. Horiz. 2020, 7, 3005 .

[34] X. Ji, Z. Li, X. Liu, H.-Q. Peng, F. Song, J. Qi, J. W. Y. Lam, L. Long, J. L. Sessler, B. Z. Tang, Adv. Mater. 2019, 31, 1902365.

[35] G. Deng, C. Tang, F. Li, H. Jiang, Y. Chen, Macromolecules 2010, 43, 1191.

[36] X. Ji, Z. Li, Y. Hu, H. Xie, W. Wu, F. Song, H. Liu, J. Wang, M. Jiang, J. W. Y. Lam, B. Z. Tang, CCS Chem. 2020, 2, 1146. 
A strategy to realize remote spatio-temporal control of luminescence behavior of materials through a photo-triggered aggregation-induced emission (PTAIE) process is reported. With the strategy, laser-mediated mask-free writing and transformable four-dimensional (4D) soft patterns of AIE-active gels are realized.

Huiling Xie, Zhao Li,* Junyi Gong, Lianrui Hu, Parvej Alam, Xiaofan Ji, Yubing Hu, Joe H. C. Chau, Jacky W. Y. Lam, Ryan T. K. Kwok,* and Ben Zhong Tang*

Photo-triggered Aggregation-Induced Emission and Direct Generation of 4D Soft Patterns

ToC figure

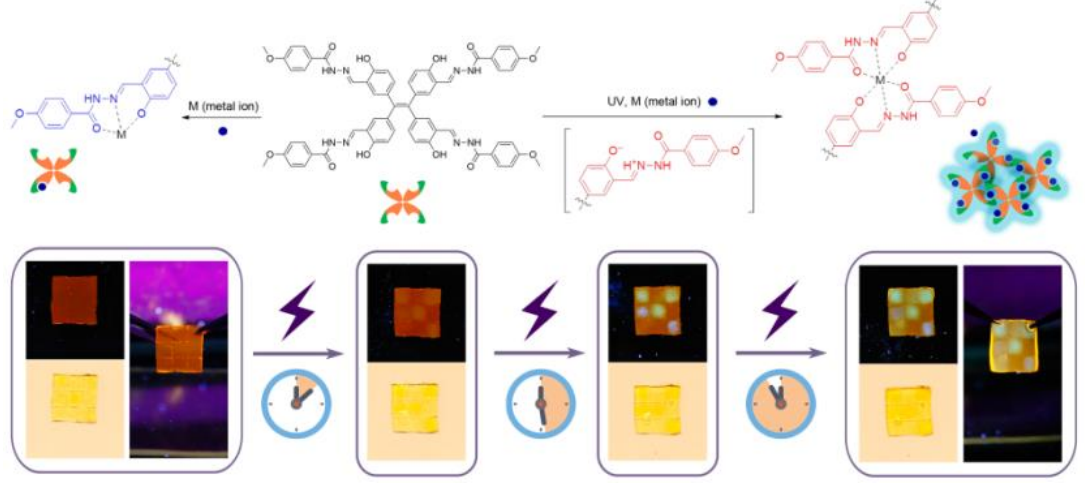

